\title{
Imaging the postoperative patient: long-term complications of gastrointestinal surgery
}

\author{
Daniel Ramos-Andrade ${ }^{1} \cdot$ Luísa Andrade $^{1} \cdot$ Catarina Ruivo $^{1} \cdot$ Maria Antónia Portilha $^{1}$. \\ Filipe Caseiro-Alves $^{1,2} \cdot$ Luís Curvo-Semedo ${ }^{1,2}$
}

Received: 24 December 2014 / Accepted: 24 November 2015/Published online: 5 December 2015

(C) The Author(s) 2015. This article is published with open access at Springerlink.com

\begin{abstract}
Objectives The objectives of this review are (1) to become acquainted with the long-term complications of surgery of the gastrointestinal tract, and (2) to appreciate the appropriate use of imaging in the assessment of long-term complications. Background Gastrointestinal tract surgery comprises a group of procedures performed for a variety of both benign and malignant diseases. In the late postoperative setting, adhesions and internal hernias are the most important complications. and they can be further complicated by volvulus and ischemia. At present, computed tomography (CT) is the workhorse for evaluating late postoperative complications. Accurate imaging assessment of patients is essential for adequate treatment planning.

Imaging findings or procedure details In this pictorial essay we will review the most frequent long-term complications after gastrointestinal surgery, including adhesions, afferent loop syndrome, closed-loop obstruction, strangulated obstruction, internal hernias, external hernias, anastomotic strictures and disease recurrence. Examples will be depicted using iconography from the authors' imaging department.

Conclusions Knowledge of the most frequent complications after gastrointestinal surgery in the late postoperative period is of paramount importance for every radiologist, so that potentially life-threatening situations can be promptly diagnosed and adequate therapy can be planned.
\end{abstract}

Luís Curvo-Semedo curvosemedo@gmail.com

1 Medical Imaging Department, Coimbra Hospital and University Centre, Coimbra, Portugal

2 Faculty of Medicine, University of Coimbra, Coimbra, Portugal
Teaching points

- Long-term postoperative complications of gastrointestinal tract surgery can be divided into procedure-related and disease-related categories.

- The most common procedure-related complications are internal hernias and adhesions.

- The most frequent disease-related complications are mainly associated with neoplastic or inflammatory recurrence.

- Computed tomography is the most useful examination when such complications are suspected.

Keywords Postoperative complications $\cdot$ Intestinal obstruction · Afferent loop syndrome · Abdominal hernia . Surgical adhesions

\section{Introduction}

An acquaintance with the types of surgery, including the most common anastomosis, and with their most frequent complications are the first steps in preventing misdiagnosis of potentially life-threatening complications following gastrointestinal surgery.

The radiologist is often faced with altered anatomic findings that hamper the ability to differentiate between an expected postoperative finding and a real complication. Therefore, communication with the referring surgeon is strongly advised in such situations before performing a diagnostic examination.

Gastrointestinal contrast studies are more commonly used to look for immediate postoperative complications, such as intestinal leak or anastomotic dehiscence.

Computed tomography (CT) is currently the workhorse for evaluating late postoperative complications, with the exception of magnetic resonance imaging (MRI) for suspected recurrence of rectal cancer or inflammatory bowel disease. 
Table 1 Long-term postoperative complications of GI tract surgery
Procedure-related:

- Herniation (internal and external)

- Adhesions

- Afferent loop syndrome

- Anastomotic strictures

Disease-related:

- Neoplastic recurrence

- Inflammatory bowel disease recurrence

Late postoperative complications can be classified as procedure- or disease-related [1] (Table 1).

Adhesions and internal hernias are the most important procedure-related complications in the late postoperative period, and can be further complicated by volvulus and ischemia. Anastomotic strictures are also relatively frequent following GI tract surgery.

Disease-related complications are typically related to disease recurrence involving both neoplastic and inflammatory conditions.

\section{Procedure-related complications}

\section{Internal hernias}

Internal hernias $(\mathrm{IH})$ are defined as the protrusion of the viscera through a normal or abnormal peritoneal or mesenteric aperture within the peritoneal cavity [2-4].

These hernias can be either congenital or acquired. The major classifications of internal hernias include paraduodenal (53\%), pericecal (13\%) and transmesenteric hernias (8\%), hernias through the foramen of Winslow (8\%) and intersigmoid hernias $(6 \%)[2,4]$.

Although paraduodenal hernias are classically regarded as the most prevalent, the incidence of transmesenteric hernias (TMH) has been growing given the increased number of operative procedures that involve Roux-en-Y surgery, such as gastric bypass surgery and liver transplants [2, 3]. In fact, IH are almost as frequent a cause of obstruction as adhesions in patients who receive liver transplants with Roux-en-Y anastomosis confection [5].

Clinically, IH can be asymptomatic, or cause nonspecific intermittent abdominal pain or full-fledged strangulated obstruction (the most common presentation). As these hernias are difficult to identify clinically, imaging plays a pivotal role in their evaluation. Herniation of the bowel loops through the defect can be a transient phenomenon that can further confound diagnosis. Clinical or imaging evaluation performed during a symptomatic period is more likely to reveal the abnormality [6].

Transmesenteric hernias are particularly prone to complications (volvulus and strangulation), and symptom onset is usually more acute than in other types of internal hernias [2, 6]. They occur in the adult population who has had abdominal surgery, especially Roux-en-Y surgery, which is now the most frequent type of acquired internal hernia according to a recent study $[2,3,6]$. Transmesenteric internal hernias can be divided into three groups: through a defect in the transverse mesocolon with retrocolic Roux limb (the most common type), through a defect in the small-bowel mesentery at the jejuno-jejunal anastomosis, and posterior to the Roux jejunal loop (Peterson defect) [7, 8].

It is important to differentiate small-bowel obstruction (SBO) due to internal hernia from obstruction due to adhesions, since the former requires emergent surgical treatment [9]. A combination of clinical and imaging criteria can help distinguish between the two. In SBO secondary to adhesions, an abrupt angulation of a bowel segment is more likely to occur. Small bowel obstruction due to IH tends to present much longer after surgery, and a collection of dilated smallbowel loops lying adjacent to the abdominal wall, without overlying omental fat and with central displacement of the adjacent colon, associated with crowding, distortion, and engorgement of mesenteric vessels, is seen on CT [7]. A cluster of loops of small bowel cephalic to the transverse mesocolon between the stomach and spleen in the left upper quadrant is typical of the transmesocolic subtype, and a group of smallbowel loops in a peripheral abdominal location is typical of the transmesenteric jejuno-jejunal type [7, 10]. The Peterson type of TMH has no distinctive findings, but because the

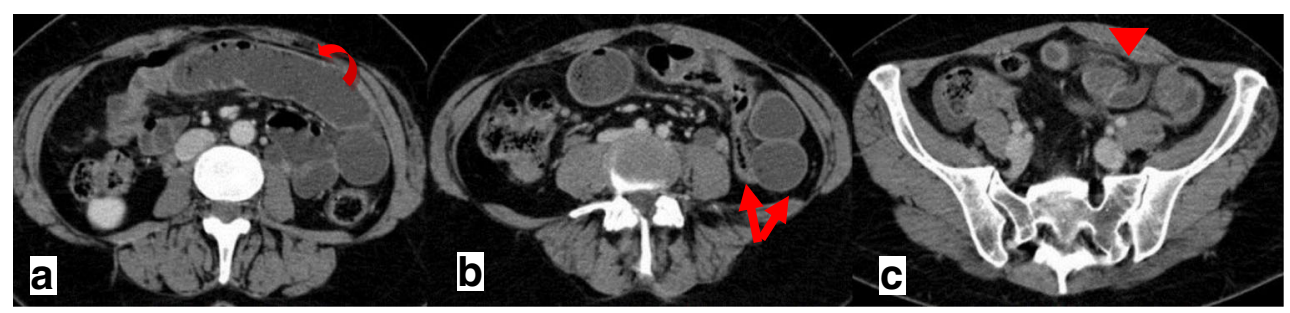

Fig. 1 a-c Surgically-proven TMH in a patient with abdominal pain. There is no fat between the fluid-filled dilated small-bowel loop and the anterior abdominal wall (curved arrow in a). The small-bowel loops are placed lateral to the descending colon with medial deviation of it (arrows in $b$ ). There is twisting of the bowel loops and mesentery-whirl sign (arrowhead in c) 


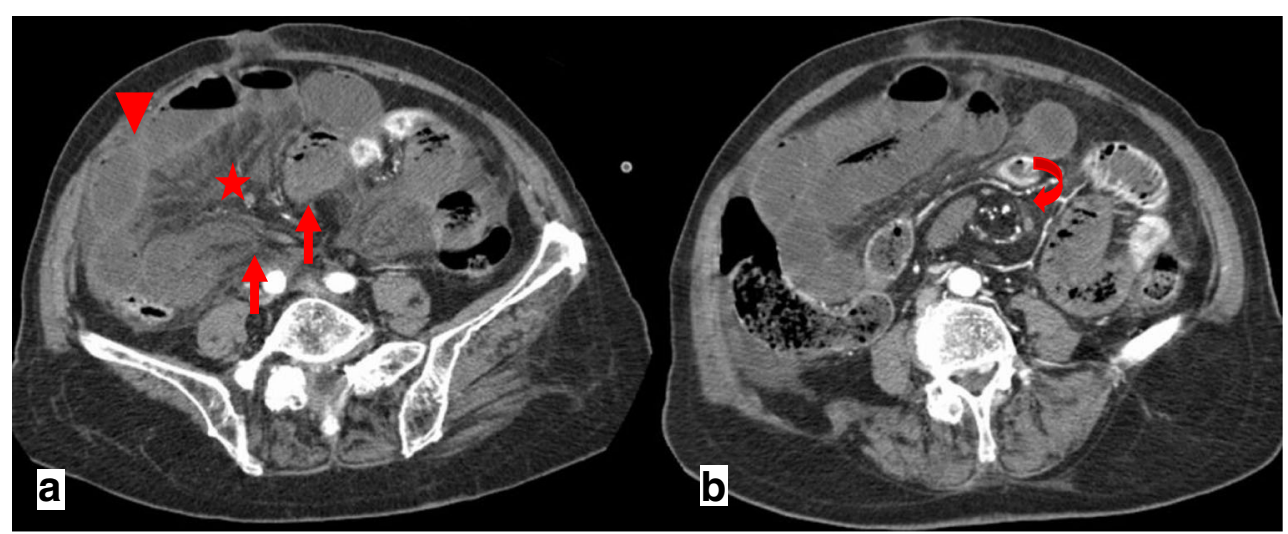

Fig. 2 a, b Patient with abdominal pain because of a closed-loop SBO. Both ends of a fluid-filled distended closed loop taper fusiformly, in a way similar to a beak - the beak sign (arrows in a). Mesenteric edema (star in a), peritoneal fluid and reduced enhancement of the bowel wall

herniation most often occurs from right to left, there may also be a cluster of dilated loops in the left upper quadrant $[2,7]$.

Multi-planar reformation (MPR) oriented to the "intestinemesentery-directed plane" and vertical to this plane should be performed in order to increase the likelihood of detection of the hernial orifice. This can be identified as an area where the mesentery of the closed loop converges on a given multiplanar view; on the plane vertical to the affected intestine, the hernial orifice shows a round/oval configuration of the converged mesentery [8].

Among other signs of internal hernias following Rouxen-Y gastric bypass surgery, which include small-bowel obstruction, clustered small-bowel loops, small bowel other than duodenum located behind the superior mesenteric artery, presence of the jejunal anastomosis to the right of the midline, and engorged mesenteric lymph nodes, the

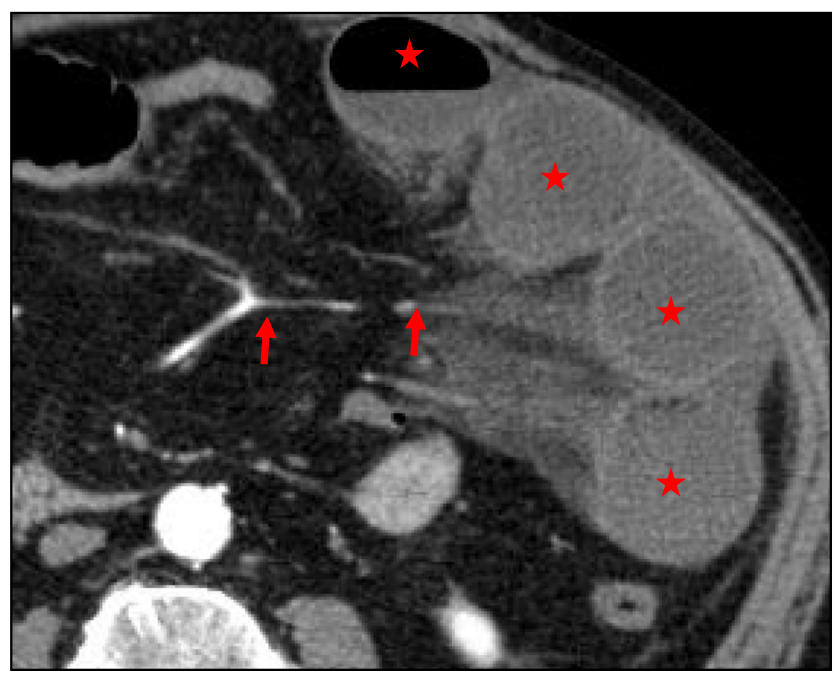

Fig. 3 Patient with closed-loop obstruction of the small bowel. Fluidfilled dilated loops of small bowel with a radial distribution (stars) and stretching of the mesenteric vessels (arrows) are seen comparatively to other small-bowel loops (arrowhead in a) are also evident, findings that suggest strangulation. A slightly cranial image shows the twisting of the mesenteric vessels at the mesenteric root—whirl sign (curved arrow in $b$ )

swirling of the mesenteric vessels is most predictive [11] (Fig. 1).

Transmesenteric hernias are generally more difficult than other IH to diagnose by CT because they lack a confining sac and thus are located potentially anywhere within the abdomen $[2,6]$.

Left-sided paraduodenal IH is the leading differential diagnosis of TMH, but has different distinctive characteristics, such as a sac-like mass of dilated bowel lateral to the ligament of Treitz that displaces and indents the transverse colon and stomach [6].

Volvulus and ischemia of the herniated small bowel are frequent complications of TMH. Typical findings of strangulated closed-loop obstruction are seen (whirl

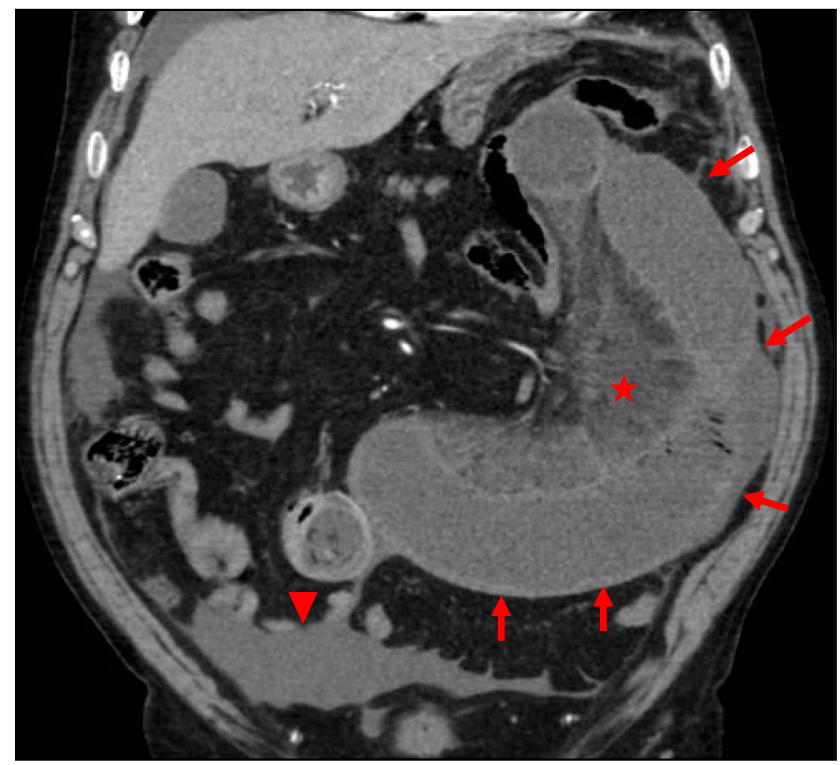

Fig. 4 Same patient as Fig. 3. Fluid-filled dilated loop of small bowel with a U/C shape configuration representing a closed-loop obstruction. There is also reduced enhancement of the bowel wall (arrows), densification of the mesentery (star) and free fluid (arrowhead) suggesting strangulation 


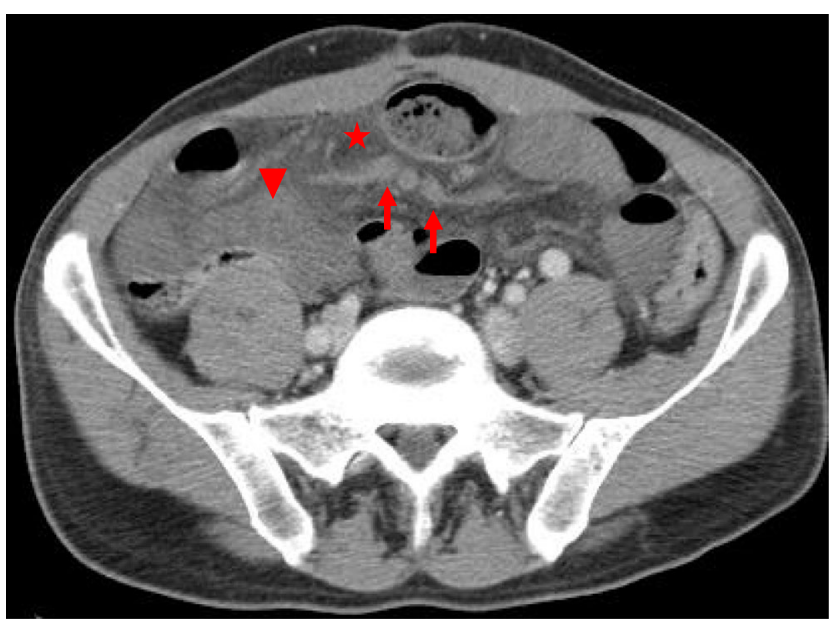

Fig. 5 Patient with strangulated obstruction. Engorged mesenteric veins (arrows) associated with mesenteric edema (star) and free fluid (arrowhead) are seen

sign, engorged blood vessels, mesenteric edema, ascites and bowel wall thickening) [6].

\section{Closed-loop bowel obstruction and incarceration}

A closed-loop bowel obstruction or incarceration is a type of mechanical intestinal obstruction in which a segment of bowel is occluded at two separate points along its length, almost always adjacent to each other, as the result of a single constrictive lesion. This anatomical configuration can lead to twisting of the loop along its long axis, and thereby produce a bowel volvulus, with subsequent compromise of the vascular supply and ischemia - strangulating obstruction. Although incarceration and strangulation are related phenomena, incarceration may occur without strangulation and may resolve spontaneously [12].

Closed-loop obstruction is most commonly caused by postoperative adhesive bands, and is less frequently due to internal or external hernias [13].

CT findings of closed-loop obstruction (incarceration) include the classical sign of mechanical obstruction (a distinct point of transition between dilated and collapsed bowel loops) and additional signs, such as:

- The beak sign, representing the beak-shaped morphology of the dilated loop toward the obstruction, when imaged in a longitudinal orientation (Fig. 2)

- The whirl sign, which corresponds to the whirled appearance of the mesenteric vessels in the middle of the obstruction point (Fig. 2). The tightness of the whirl pattern reflects the degree to which the mesentery and vessels are rotated. Although hampered by low sensitivity ( $60 \%$ ), a recent study has shown that if this sign is present on a CT scan, a patient is 25 times as likely as a patient without it to undergo surgery $[13,14]$.

- A radial distribution, which refers to stretched mesenteric vessels converging toward the central point of obstruction (Fig. 3).

- The U/C sign, which refers to the shape of the incarcerated distended loop (Fig. 4). Although it has been described on axial images when the loop is horizontally oriented, it can also be well depicted on sagittal and coronal reformations when the loop is vertically oriented [13].

Multiplanar reformatted images may prove decisive in achieving correct diagnosis of a closed-loop obstruction [13].

CT findings of strangulated obstruction include wall thickening, altered pattern of enhancement of the bowel wall, dilated mesenteric veins, mesenteric edema, ascites, pneumatosis intestinalis, pneumatosis portalis and pneumoperitoneum.

The most common CT finding in bowel ischemia, although nonspecific, is bowel wall thickening. It is caused by mural edema (low attenuation before contrast administration), hemorrhage (high attenuation before contrast administration) and/ or superinfection of the ischemic bowel wall. If unenhanced $\mathrm{CT}$ is not performed prior to contrast-enhanced $\mathrm{CT}$, the ability to differentiate between intramural hemorrhage and hyperemia and/or hyperperfusion is impaired [15].

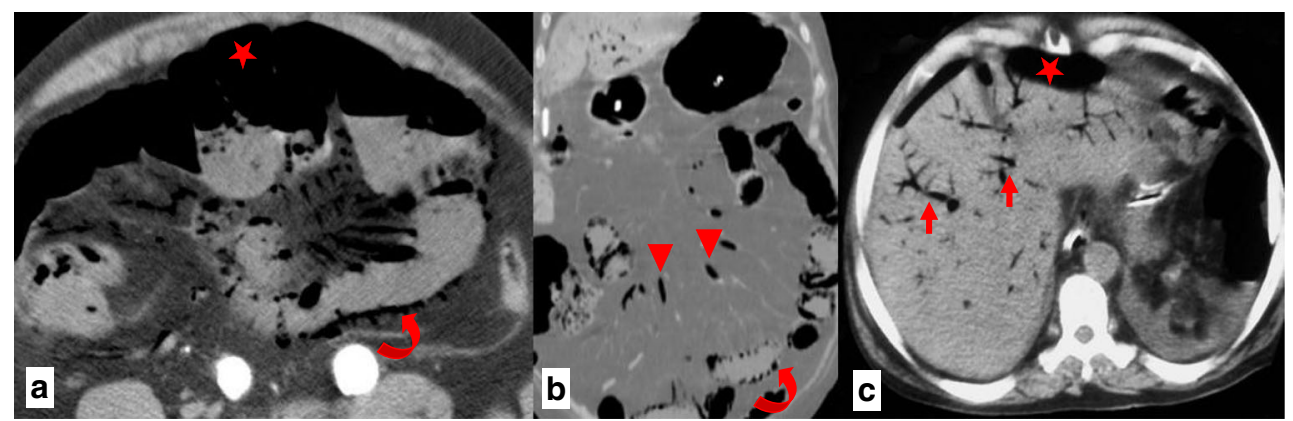

Fig. 6 a-c Patient with bowel ischemia secondary to a strangulated obstruction. Gas inside the mesenteric veins (arrowheads in $b$ ) coming from a bowel segment that also presents intramural gas (curved arrows in $a, b)$ after perforation of a gangrenous bowel wall are seen. Portal venous gas (arrows in c) and pneumoperitoneum (stars in $a, c)$ are also evident 
Fig. 7 a, b Patient with an incisional external hernia. A cluster of fluid and air-filled dilated bowel loops is found inside the hernial sac on the right flank (stars). Narrowing of the afferent and efferent ends of the closed loop at the site of hernial neck can also be appreciated - the beak sign (arrows in a).

Stretching of the mesenteric vessels is seen on the coronal reformation (arrowhead in $b$ )

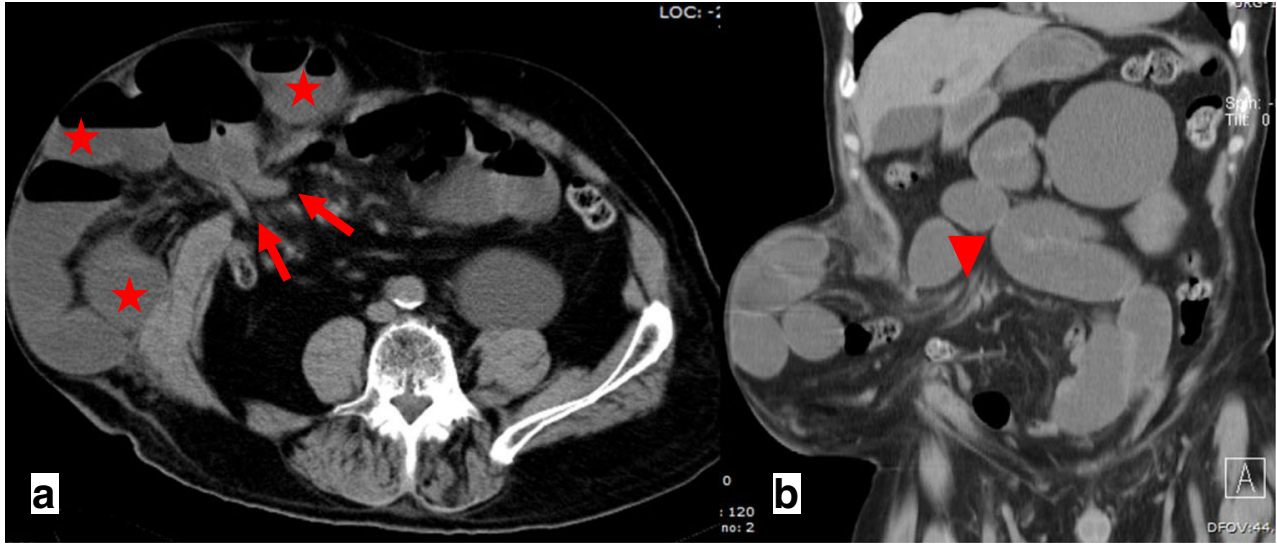

On contrast-enhanced CT, a highly specific but less sensitive finding is absent or diminished parietal contrast enhancement. In some cases, prolonged enhancement due to delayed return of the venous blood, with subsequent slowing of the arterial supply, can also be seen [16].

As ischemia advances, venules in the mesentery become engorged with blood. Mesenteric fat stranding due to edema and/or ascites is also a nonspecific CT finding in acute bowel ischemia (Figs. 4 and 5). In a recent prospective study, fluid in the mesentery adjacent to abnormal (thickened, dilated or both) bowel loops was the individual sign most frequently associated with strangulation (88 \%) and had an extremely high negative predictive value [17].

When bowel wall thinning rather than thickening occurs, transmural small-bowel infarction has probably already ensued. In this setting, it is also more likely for pneumatosis intestinalis, pneumatosis portalis and pneumoperitoneum to develop (Fig. 6). Pneumatosis intestinalis is caused by dissection of luminal gas into the bowel wall across the compromised mucosa. Portomesenteric venous gas is due to the propagation of that gas to the mesenteric venous system, towards the liver. Free intraperitoneal air represents perforation of the gangrenous bowel loop $[15,16,18]$.

Clinical and laboratory differentiation between simple obstruction and incarceration/ strangulation is very difficult, and treatment delay is a major prognostic factor for increased morbidity and poor survival. CT is extremely useful in discriminating between these two entities, and it plays an instrumental role in determining appropriate patient treatment (medical versus surgical). Findings of
Fig. 8 a Type 0 "parastomal hernia": the peritoneum follows the wall of the bowel, with no sac formation (arrows). b Type Ia "parastomal hernia": only the bowel forming the colostomy is seen, with a sac $<5 \mathrm{~cm}$ (arrows). c Type Ib parastomal hernia: the hernial sac contains only the bowel loop forming the colostomy, but its width is larger than $5 \mathrm{~cm}$ (arrows). d Type II parastomal hernia: there is omentum (star) within the hernial sac (arrows). e Type III parastomal hernia: there are bowel loops (arrowhead) other than the one forming the stoma (arrows) at the right flank; note also an incisional hernia at the midline in the umbilical region (curved arrow)

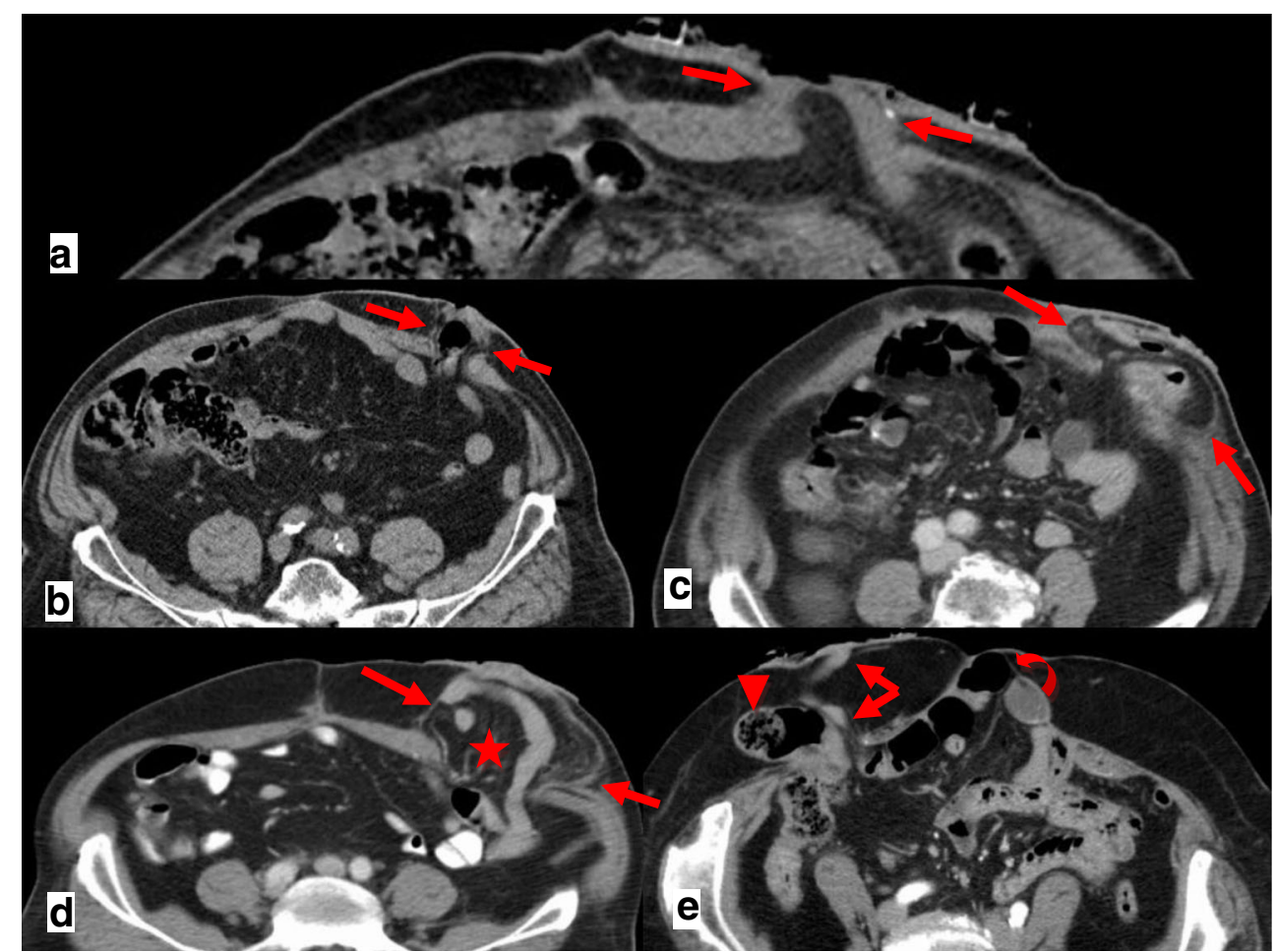


Table 2 Parastomal hernia CT classification

$\begin{array}{ll}\text { Type } 0 & \begin{array}{c}\text { Peritoneum follows the wall of the bowel, with no sac } \\ \text { formation }\end{array} \\ \text { Type Ia } & \text { Only bowel forming the colostomy, with a sac }<5 \mathrm{~cm} \\ \text { Type Ib } & \begin{array}{c}\text { Hernial sac containing only bowel forming the colostomy, } \\ \text { with a sac }>5 \mathrm{~cm}\end{array} \\ \text { Type II } & \begin{array}{l}\text { Hernial sac containing omentum } \\ \text { Type III }\end{array} \\ & \begin{array}{l}\text { Hernial sac containing intestinal loop other than the } \\ \text { bowel forming the stoma }\end{array}\end{array}$

closed-loop obstruction should result in close monitoring, and if clinical symptoms persist, early surgery is suggested. In patients in whom signs of strangulation are already evident, emergency surgery is mandatory [12].

\section{External hernias}

An incisional hernia is a type of external hernia caused by an incompletely healed surgical wound. Typically, peritoneal fat, with or without incorporation of bowel loops, protrudes through the hernial defect (Figs. 7 and 8e).

Stomal and parastomal hernias are a subtype of incisional hernias that involve herniation of the bowel or the mesentery at the site of or adjacent to a stoma [19]. Even after closure of the enterostomy, the ostomy site can remain a potential area of herniation [20]. Most parastomal hernias occur within 8 months of surgery. The etiology is multifactorial and includes characteristics such as obesity, malnutrition, increased intraabdominal pressure and chronic respiratory disease [21].

Parastomal hernias are quite common, with an expected prevalence of $33-78 \%$, depending on clinical or CT

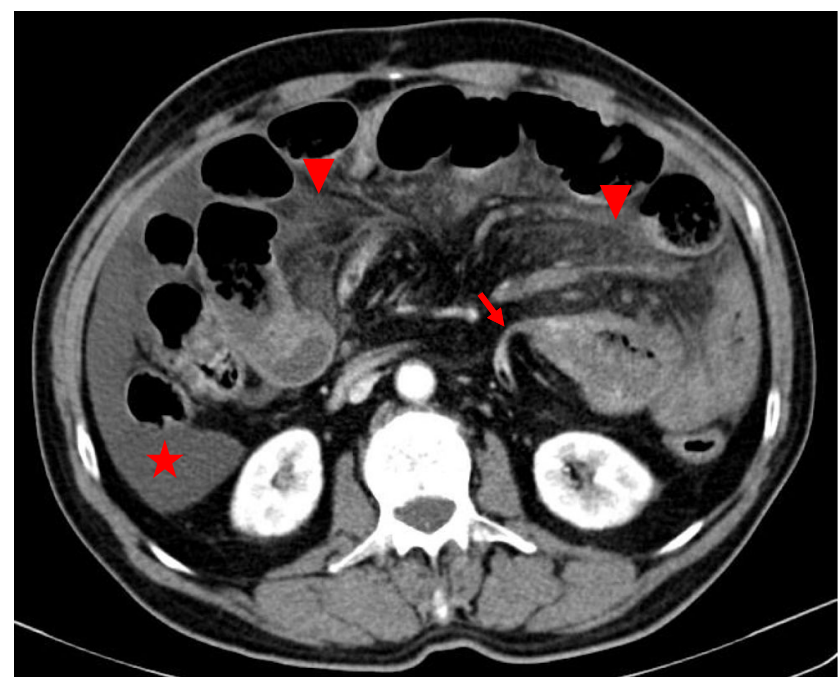

Fig. 10 Small-bowel dilation due to an adhesion in a patient with abdominal pain. There is an abrupt narrowing and acute angulation of the loop, with no identifiable underlying lesion (arrow). There is also ascites (star) and mesentery densification due to edema (arrowheads), findings suggestive of strangulated obstruction

evaluation. However, unless obstruction, perforation or stoma malfunction occur, there is no need for surgical repair [19-22].

A recent study suggested a CT classification for parastomal hernias according to the possible contents of the hernial sac, as follows: type I (hernial sac containing the stoma loop), type II (sac containing omentum), type III (sac containing a bowel loop other than stoma) (Table 2). Type 0 and Type Ia are considered normal findings and not true hernias [21] (Fig. 8).

Incarceration and strangulation can supervene with incisional hernias, as with any other abdominal wall hernias
Fig. 9 a, b Abdominal CT of a patient with obstructive symptoms. There is diffuse fluid dilation of small-bowel loops (arrowheads in $b$ ) proximal to a small segment of dilated small bowel incarcerated at the hernial sac (arrow in a). The presence of fat stranding and a moderate amount of free fluid at the site (curved arrows in $a, b$ ) should raise suspicion of a strangulated hernia

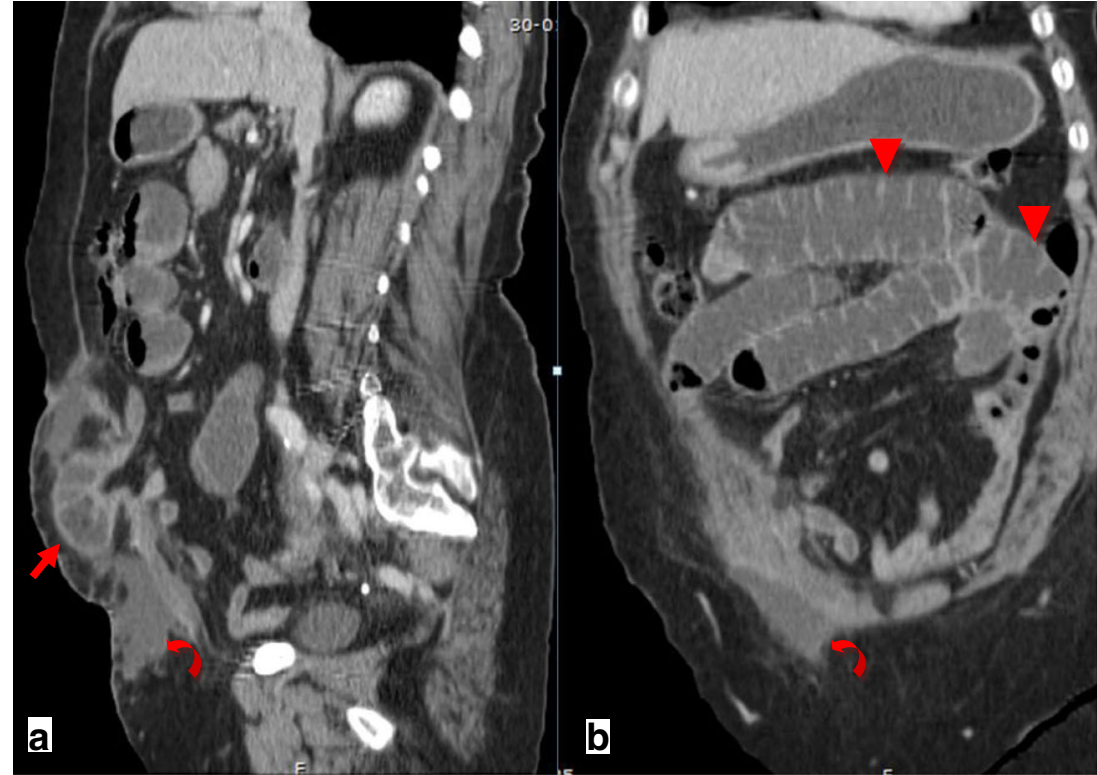


Fig. 11 a, b Adhesion-related SBO in a patient with acute abdominal pain. Dilated smallbowel loops closely applied to the anterior peritoneum (stars in $a$ ), stretching of the bowel loop (curved arrow in a) and traction deformities of the bowel loop (arrow in b) are seen

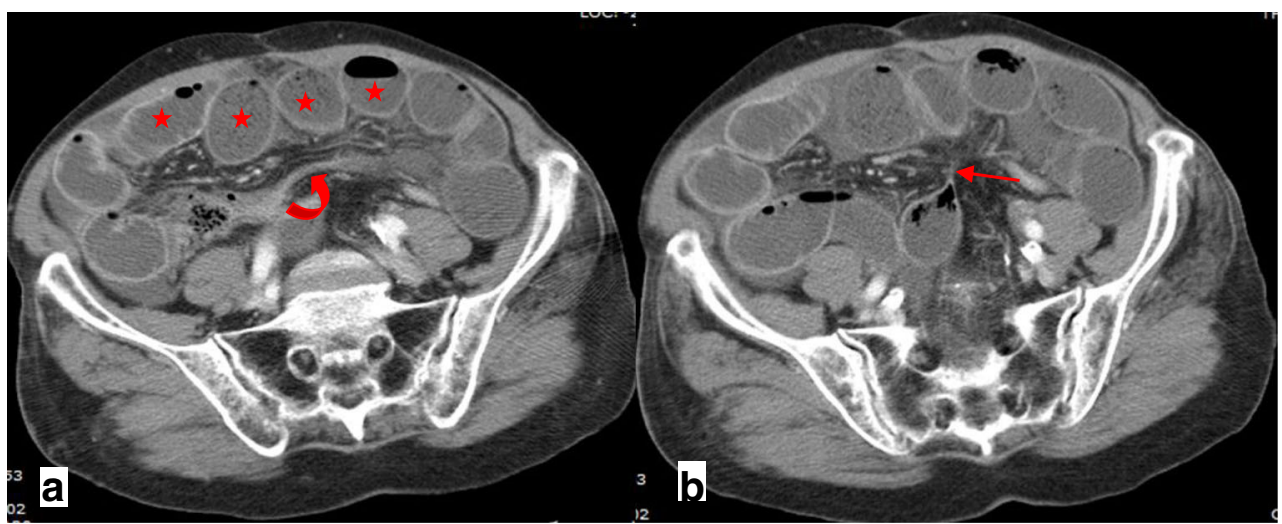

elsewhere. Strangulation occurs with typical findings as described above, such as bowel wall thickening, free fluid and fat stranding [19] (Fig. 9).

\section{Adhesions}

Adhesive bands are funiculate structures that form between the peritoneum of tissues and organs, often as a result of injury during surgery. They are composed of fibrous tissue and abundant fat [8].

Adhesions are the most common cause of bowel obstruction after surgery, and they constitute the leading cause of long-term reoperation following abdominal and pelvic surgery.

Bowel loops that pass through the orifice between the adhesive band and the peritoneum form a type of internal hernia that can evolve to closed-loop obstruction. CT findings in this situation are similar to those in other internal hernias (cf. "Internal hernias"). Overt obstructive small-bowel disease related to adhesions can be further subdivided into simple obstruction and closed-loop or strangulated obstruction [23].

Nevertheless, a non-obstructive subtype can also be found. These patients may have intermittent or lowgrade small-bowel obstruction, for which $\mathrm{CT}$ has poor
Fig. 12 Patient with chronic abdominal pain and small-grade bowel obstruction. A fat density band is seen crossing the bowel loop in both the coronal and axial planes - fat-bridging sign (circles)

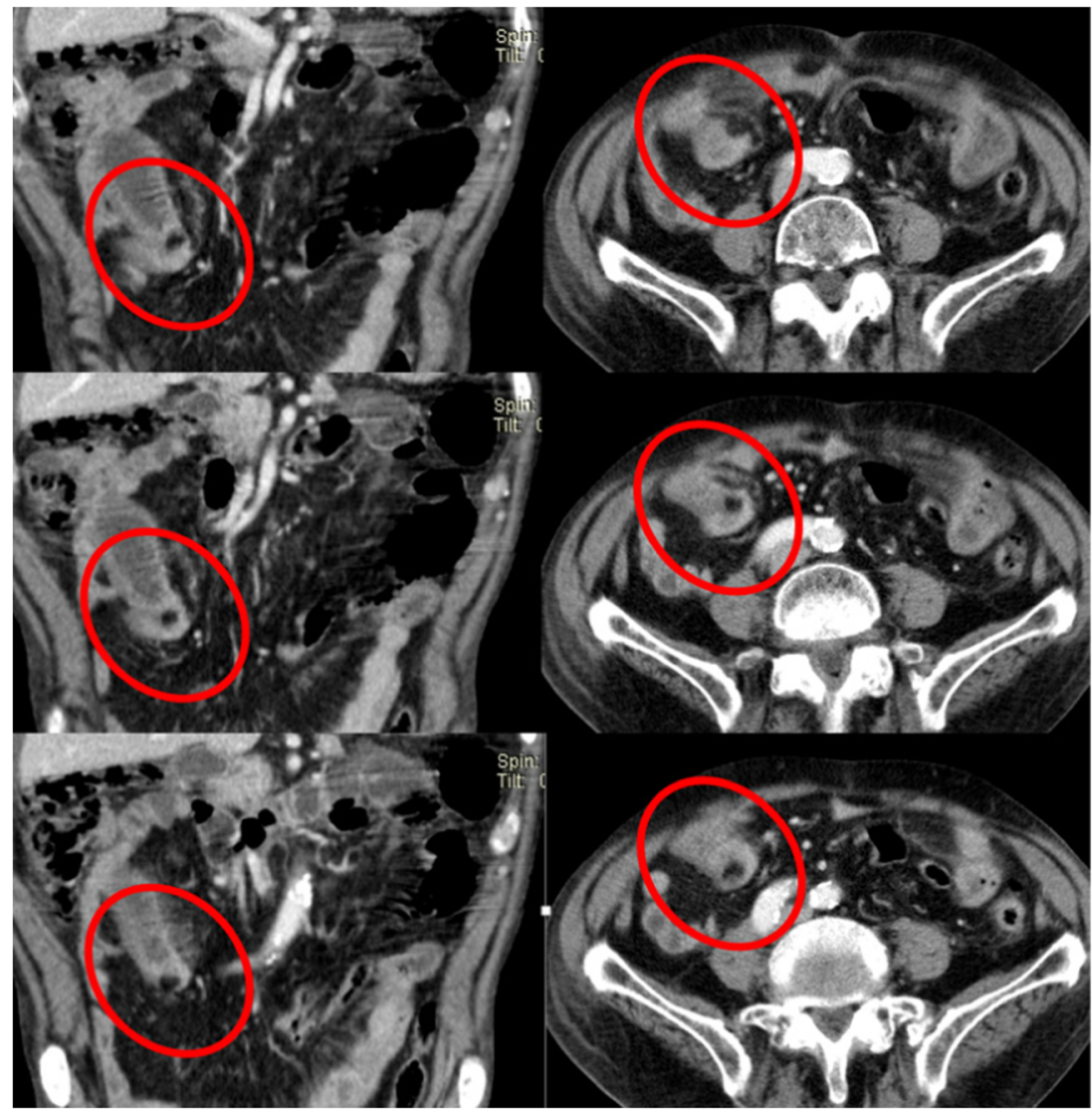


Fig. 13 Upper gastrointestinal series of a patient with abdominal pain and vomiting who had undergone partial gastrectomy with Billroth II reconstruction following gastric adenocarcinoma 9 years earlier. There is nonfilling of the afferent loop (star) and a filling defect at the location of the anastomosis (arrows)

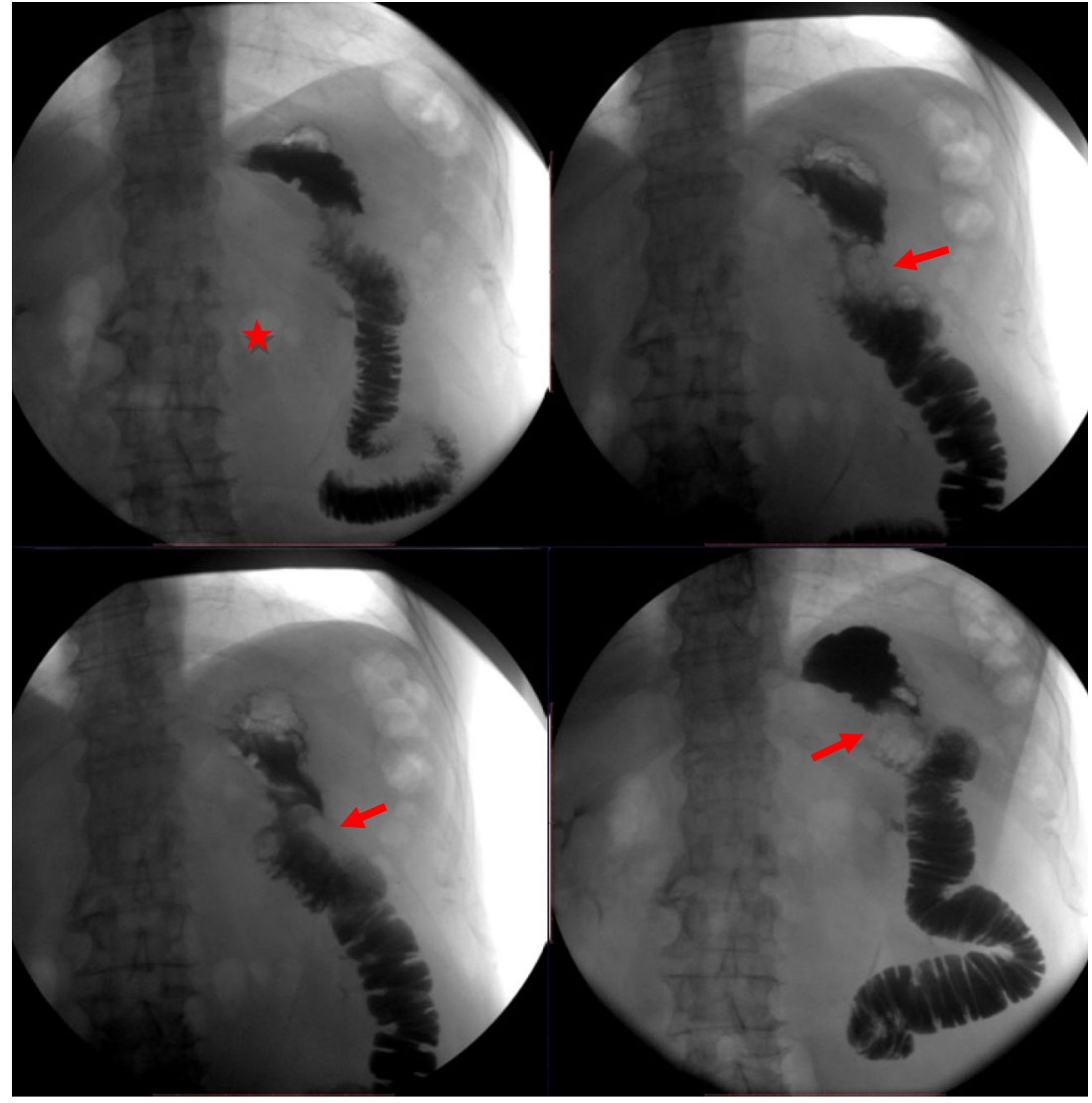

sensitivity. CT enteroclysis or dynamic MRI may be important options for better demonstrating the transition point [8, 23].

The diagnosis of small-bowel obstruction due to adhesions is presumed when all other causes of obstruction have been ruled out on CT. The main CT findings that suggest adhesions as the culprit of the obstruction include a narrow zone of transition without an identifiable lesion (such as a mass, wall thickening or adenopathy), acute angulation of the smallbowel loops, traction deformities, stretching of the bowel loops, small-bowel loops closely applied to the anterior abdominal wall and the "fat-bridging sign" which represents the adhesive band itself as a cord-like structure containing mesenteric fat that bridges two peritoneal surfaces [8, 9] (Figs. 10, 11, and 12).

Small-bowel obstruction caused by adhesions almost always requires surgery. When intestinal strangulation supervenes, immediate abdominal surgery is mandatory. However, the risk of additional adhesions increases as the number of surgeries increases.

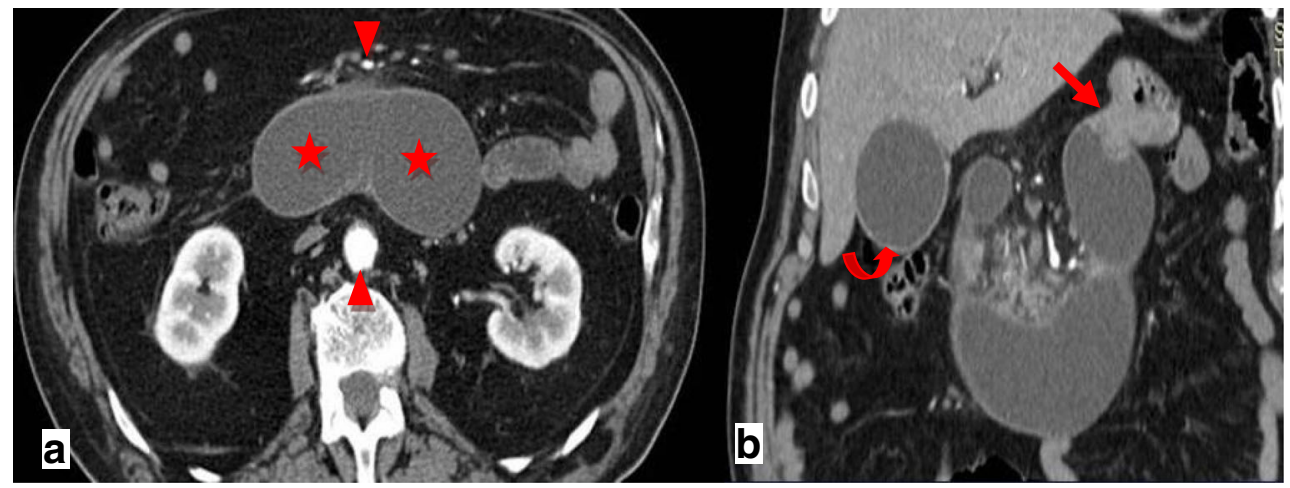

Fig. 14 a, b Abdominal CT of the same patient as in Fig. 13. A fluidfilled tubular structure (stars in $a$ ) crossing the midline between the aorta and the superior mesenteric vessels (arrowheads in a) is seen, with associated gallbladder distension (curved arrow in b)-afferent-loop syndrome caused by tumor recurrence, depicted as an irregular enhancing mass at the anastomosis site (arrow in $b$ ) 


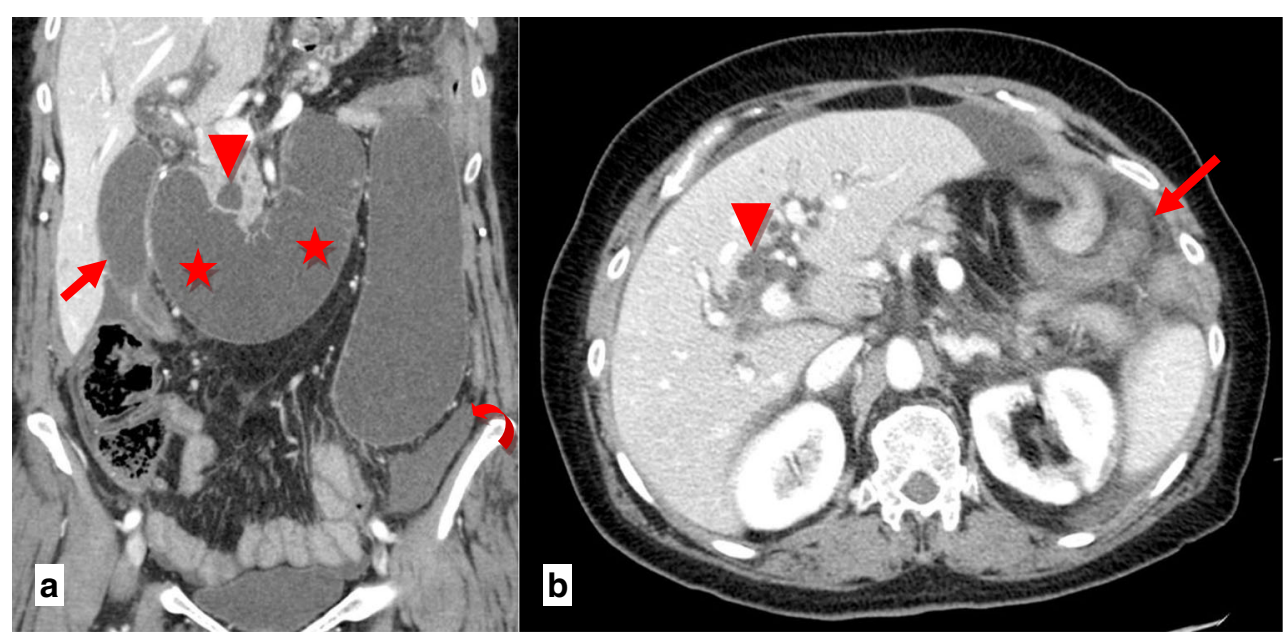

Fig. 15 a, b Abdominal CT of a patient with progressively worsening abdominal pain, nausea and vomiting who had undergone Billroth II partial gastrectomy for gastric carcinoma 5 years earlier. There is marked fluid-filled dilation of the duodenum (stars in a), associated with gallbladder distension (arrow in a) and intrahepatic and

\section{Afferent loop syndrome}

Afferent loop syndrome (ALS) represents mechanical obstruction of the afferent loop, occurring in $0.3-2 \%$ of gastroenterostomies, both in Billroth II surgery (the duodenum is the afferent loop) and Whipple or Roux-en-Y procedures (the Roux segment is the afferent loop) [9, 24, 25].

Most cases of ALS are due to adhesions, internal hernia, anastomotic stricture or recurrent tumor [9]. It can also be secondary to preferential gastric emptying into the afferent loop due to abnormal surgical anastomosis or because of efferent loop obstruction resulting in fluid accumulation at the afferent loop $[24,26]$. The back pressure from the dilated afferent loop can cause biliary dilatation and acute pancreatitis [26].

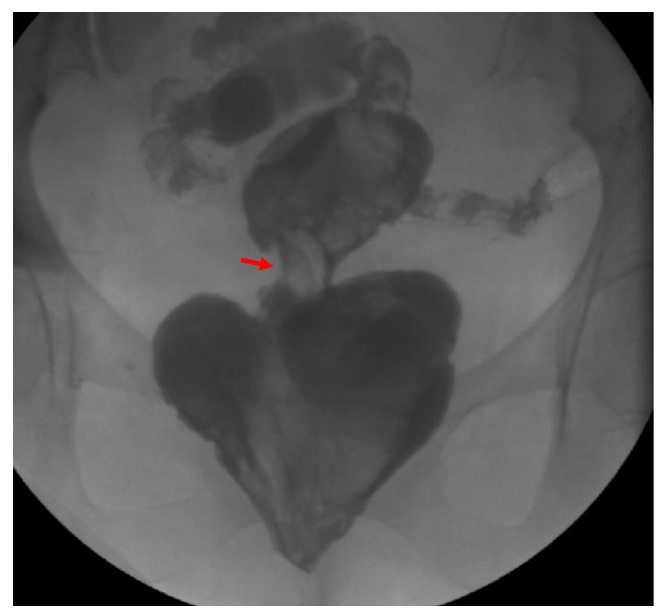

Fig. 16 Water-soluble enema shows a short anastomotic stricture (arrow) and proximal dilatation in a patient who had undergone anterior resection of the rectum some months earlier extrahepatic biliary dilation (arrowheads in $a, b$ ), caused by an internal hernia with volvulus as shown by the twisted configuration of the bowel loops at the left upper quadrant (arrow in b). The ascites (curved arrow in a) suggests supervened ischemia

The diagnosis may not be clinically suspected, as ALS may present many years after the initial surgery [27].

Although gastrointestinal contrast studies can be helpful in the diagnosis of this condition by showing non-filling of the afferent loop, non-obstructed afferent loops are not normally filled in $20 \%$ of cases. Preferential filling and retention of oral contrast in a dilated afferent limb for at least $60 \mathrm{~min}$ is another finding consistent with afferent loop syndrome [24, 26] (Fig. 13).

CT is the most important imaging tool in establishing the diagnosis and determining the site, degree and cause of ALS. A fluid-filled tubular or C-shaped structure containing small air bubbles in the right upper quadrant or crossing the midline between the aorta and the superior mesenteric vessels, with valvulae conniventes projecting into the lumen, in symptomatic patients after gastroenterostomy is characteristic (Fig. 14). Recognition of these distinctive findings should prevent the misdiagnosis of a pancreatic pseudocyst [24, 27]. The coronal plane is most helpful in detecting the causes of ALS so that adequate treatment can be chosen [24]. Complications of ALS such as biliary dilatation, pancreatitis or strangulation are also readily identified on CT [26] (Fig. 15).

\section{Anastomotic strictures}

The incidence of anastomotic strictures depends on the type of surgery performed. The causes for the stricture are not yet clearly understood. For example, in gastric bypass patients, it is thought that the period of time during which the anastomosis is exposed to an inappropriately large volume of gastric acid results in ongoing inflammation, ulceration and stricture formation [28]. Several studies also suggest an increase in the incidence of strictures with the use of stapler versus handsewn 
Fig. 17 a, b Patient with complete lower intestinal obstruction who had undergone anterior resection of the rectum some years earlier. Water-soluble enema showed no progression of contrast beyond the coloanal anastomosis (arrow in a). Pelvic $\mathrm{CT}$ revealed diffuse wall thickening (arrows in $b$ ) of the coloanal anastomosis (note the surgical staples) due to a histologically proven fibrotic stenosis
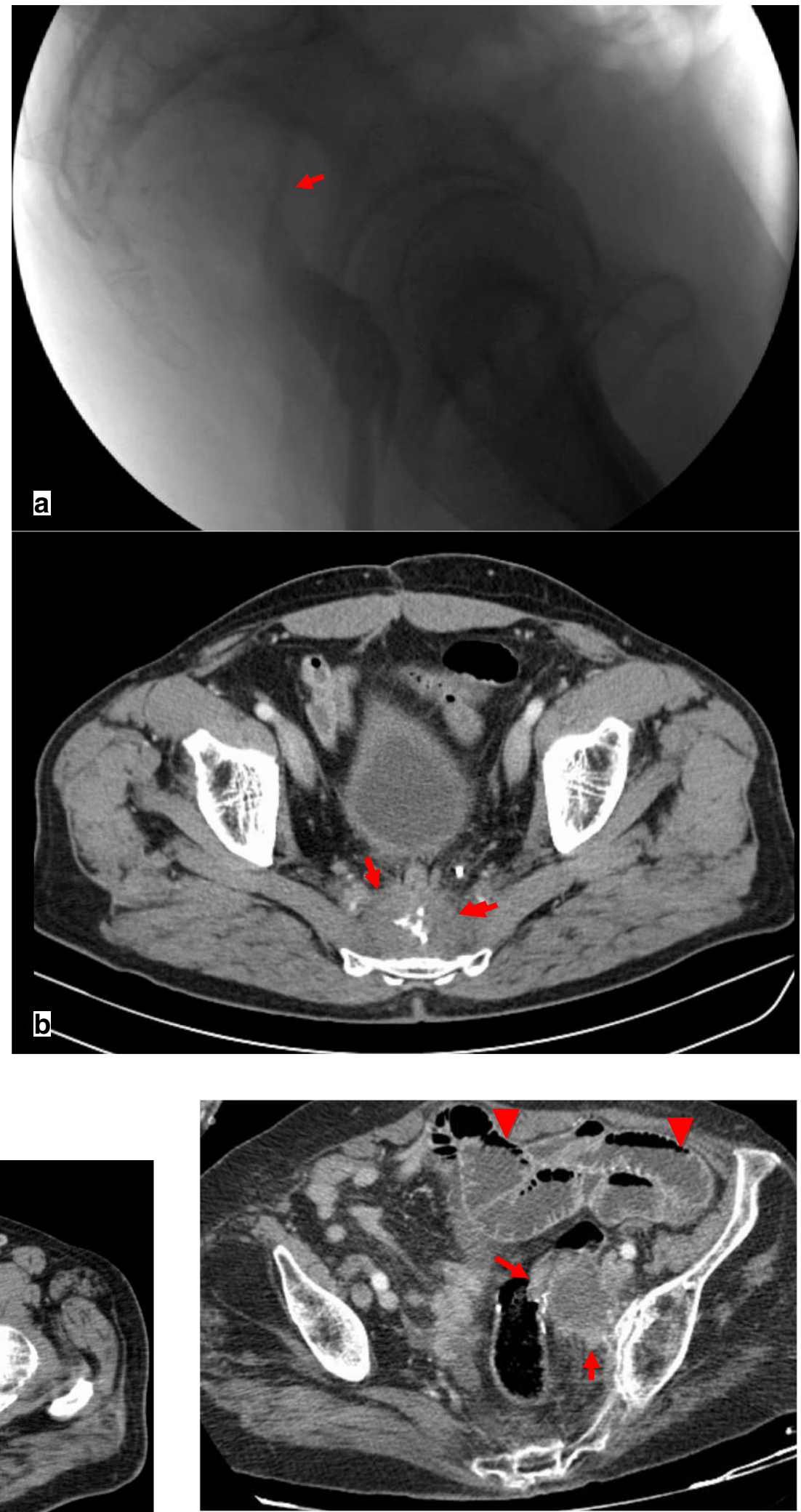

Fig. 19 Contrast-enhanced pelvic CT of a 79-year old woman with obstructive symptoms who had undergone sigmoidectomy for adenocarcinoma 1 year earlier. There is asymmetric thickening of the bowel wall and a hypodense mass adjacent to the colorectal anastomosis (arrows), causing proximal bowel distension (arrowheads), confirmed as tumor recurrence at surgery 
Fig. 20 Thoracic-abdominal CT of a patient who had undergone total gastrectomy for adenocarcinoma some years earlier. There is evidence of a soft tissue mass next to the surgical anastomosis, encircling the descending aorta (arrows) and corresponding to a non-resectable neoplastic recurrence

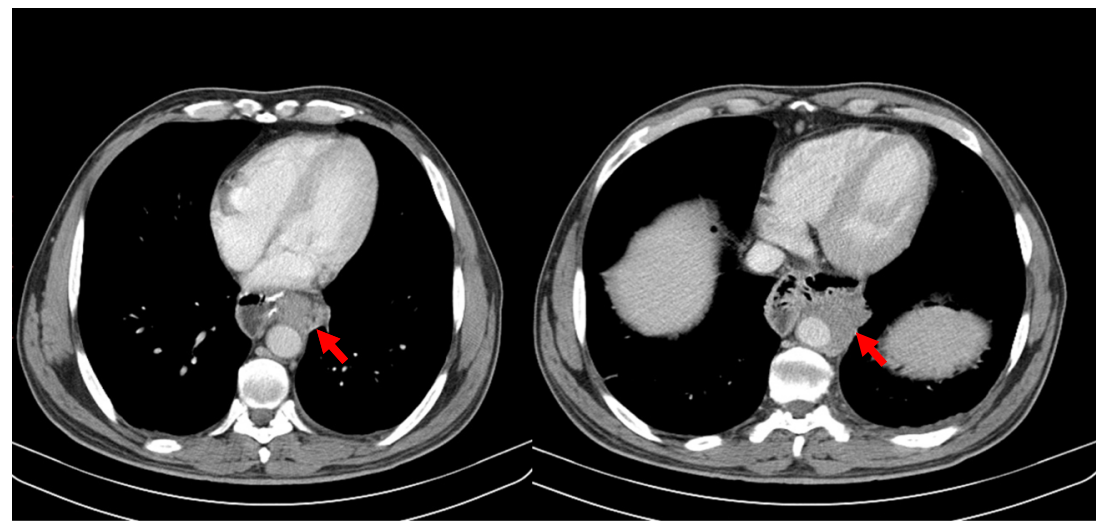

anastomosis, both for gastrojejunostomy and colorectal anastomosis $[1,29]$. For ileal pouch anal anastomosis, a metaanalysis found an incidence of anastomotic strictures of $9.2 \%[30]$.

The resulting symptoms reflect the degree of luminal stenosis, and include abdominal pain, distention, nausea and vomiting.

Gastrointestinal contrast studies are the classical method for diagnosing anastomotic strictures. They allow a clear depiction of the stenosis with delayed passage of contrast material, pre-stenotic bowel distension and possible perianastomotic fistulae (Figs. 16 and 17a).

$\mathrm{CT}$ has become increasing important when an anastomotic stricture is being considered, and is now the preferred imaging modality in this particular setting, since it is able to demonstrate not only the anastomotic abnormality but extra-wall structures as well [1]. CT findings include focal bowel wall thickening at the anastomosis and distended proximal bowel loops filled with fluid or desiccated stool [31] (Fig. 17b).

Depending on the location, strictures can be treated with manual/endoscopic dilation or surgery [31].

\section{Disease-related complications}

\section{Neoplastic recurrence}

Tumor recurrence can be the cause of obstruction several months or years after surgery.

CT is the modality of choice in this setting, as it clearly shows an enhancing mass or asymmetric wall-thickening near the site of the initially resected tumor.

Most patients who undergo abdominal perineal resection or anterior resection of the rectum will present an ill-defined presacral midline mass 3 to $5 \mathrm{~cm}$ in diameter that decreases in size with time (although it can persist indefinitely) and becomes progressively more distinct in serial imaging - fibrosis / granulation tissue - a finding that can easily be mistaken for tumor recurrence [19] (Fig. 18).

In contrast, tumor recurrence manifests as a welldefined soft tissue mass that grows on serial imaging and becomes ill-defined as it becomes more infiltrative [19] (Figs. 19 and 20).
Fig. 21 a, b CT enterography of a patient with Crohn's disease who had previously undergone segmental enterectomy. Note the surgical material at the ileal-ileal anastomosis (curved arrows in a, b). There is a fistulous tract between the thickened ileal segment near the anastomosis and the duodenum (arrows in a). MIP coronal reformation $(b)$ shows mesenteric engorgement (stars in b) and lymphadenopathy (arrow in $b$ ) in the vicinity of a thickened bowel wall segment (arrowheads in $b$ )

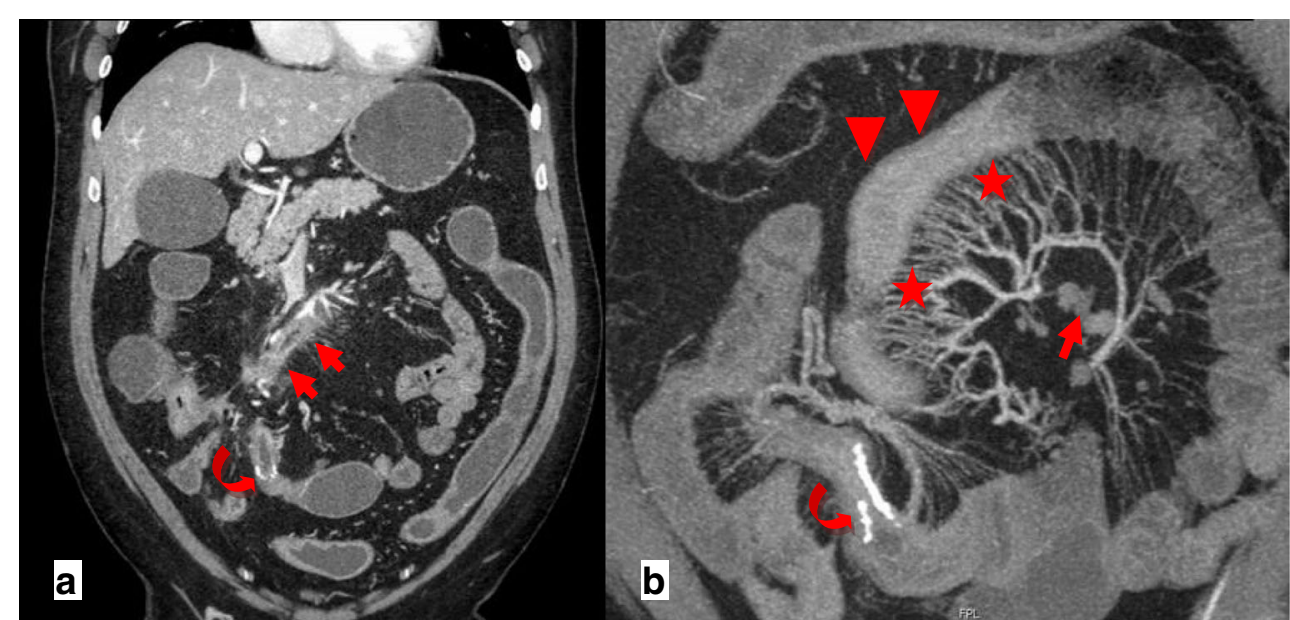


Fig. 22 A fluid collection with a thickened, hyper-enhancing wall corresponding to an abscess (star) is seen anteriorly to a bowel wall segment with thick walls at the level of the neo-terminal ileum (arrows) in a patient who had already undergone surgery due to Crohn's terminal ileitis

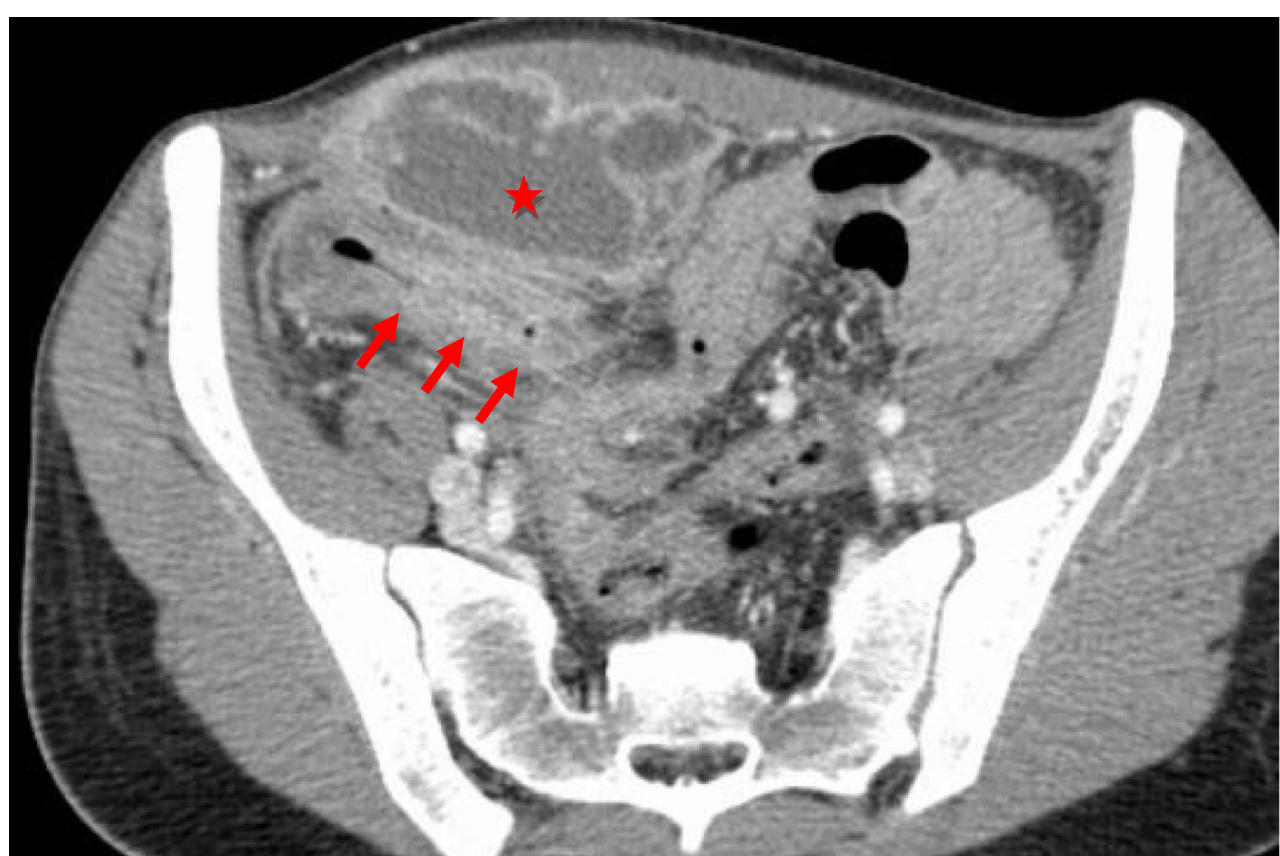

\section{Inflammatory bowel disease recurrence}

\section{Crohn's disease}

Most patients with Crohn's disease (CD) require surgery at some point in their lives, and can develop relapses either at the intestinal anastomosis or far from it, at any other segment of the GI tract. These relapses can occur in the form of new strictures, fistulae or abscesses [1].

A combination of symptom assessment plus endoscopy is still the gold standard for assessment of recurrence in postoperative CD patients [32].

$\mathrm{CT}$ enterography/enteroclysis should be the first radiologic procedure performed in patients with acute symptoms when recurrence is suspected. MR enterography/enteroclysis is increasingly being considered as the first-choice examination in acute exacerbation in a child or young adult with known CD, because it is a radiation-sparing technique in a patient who will likely be subjected to multiple serial examinations [33]. MRI is also the technique of choice for the diagnosis and characterization of perianal fistulae.

The ability to directly demonstrate the bowel wall, adjacent organs, mesentery and retroperitoneum renders $\mathrm{CT}$ and $\mathrm{MR}$ superior to any other examination in diagnosing the complications of $\mathrm{CD}$. Both methods can evidence hyper-enhancing bowel wall thickening, mesenteric edema, prominent mesenteric vessels (the "comb sign") and lymphadenopathy, as well as possible fistulae or abscesses (Figs. 21 and 22).

Although CT is by far the most frequent examination undertaken in this setting, we cannot simply discard gastrointestinal contrast studies just yet. A recent study found small bowel follow-through (SBFT) to be more sensitive and specific than $\mathrm{CT}$ for detecting recurrent $\mathrm{CD}$ in the neo-terminal ileum because of its ability to depict aphthoid ulcers not detectable on CT. Other findings of Crohn's recurrence at SBFT are luminal narrowing, thickened folds and deep ulcerations ("rose thorn" ulcers) [34] (Fig. 23).

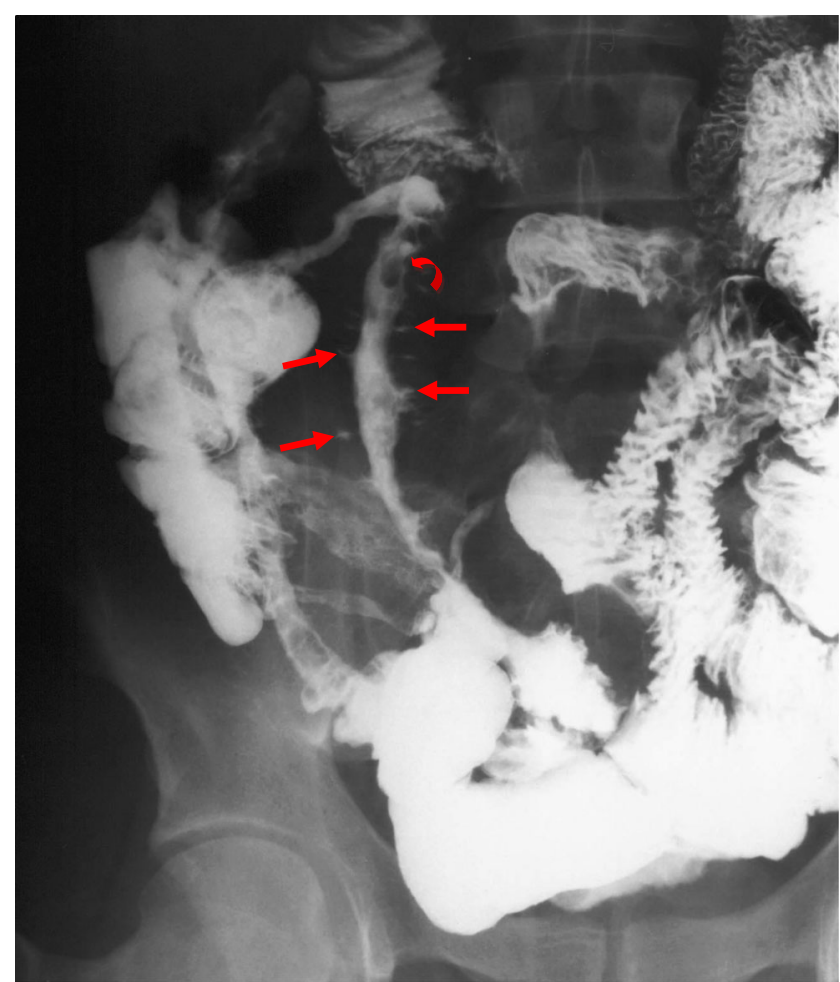

Fig. 23 Barium follow-through of a patient with active Crohn's disease: there is severe irregular narrowing of the neo-terminal ileum, with separation from adjacent bowel loops and with some areas of cobblestoning (curved arrow) and linear deep ulcers (arrows) 


\section{Ulcerative colitis}

Ulcerative colitis is normally continuous from the rectum to the colon. Total proctocolectomy is the definitive treatment for the disease, but two other types of surgery are usually performed to obviate the disadvantages of a permanent colostomy.

Patients who receive total colectomy with ileorectal anastomosis are at risk for disease recurrence at the rectum, and thus clinical and endoscopic follow-up are advised. When proctocolectomy with ileal-anal pouch is chosen, there is an increased probability of the patient developing pouchitis (ileal pouch inflammation). The diagnosis depends on clinical, endoscopic and histopathological data, and there is usually no role for diagnostic imaging in this setting [1].

\section{Conclusions}

In conclusion, knowledge of the normal appearance of the abdomen and pelvis after GI tract surgery is mandatory for every diagnostic radiologist, particularly in an emergency setting. An acquaintance with the most frequent complications in the late postoperative period, such as bowel obstruction due to internal or external hernias, adhesions, afferent loop syndrome and disease recurrence, is of paramount importance.

Communication with the referring surgeon can be a valuable adjunct for achieving diagnosis in potentially lifethreatening situations so that adequate therapy can be planned.

Open Access This article is distributed under the terms of the Creative Commons Attribution 4.0 International License (http:// creativecommons.org/licenses/by/4.0/), which permits unrestricted use, distribution, and reproduction in any medium, provided you give appropriate credit to the original author(s) and the source, provide a link to the Creative Commons license, and indicate if changes were made.

\section{References}

1. Scardapane A, Brindicci D, Fracella MR, Angelelli G (2005) Post colon surgery complications: imaging findings. Eur J Radiol 53(3): 397-409

2. Martin LC, Merkle EM, Thompson WM (2006) Review of internal hernias: radiographic and clinical findings. AJR Am J Roentgenol 186(3):703-717

3. Hong SS, Kim AY, Kim PN, Lee MG, Ha HK (2005) Current diagnostic role of $\mathrm{CT}$ in evaluating internal hernia. J Comput Assist Tomogr 29(5):604-609

4. Takeyama N, Gokan T, Ohgiya Y, Satoh S, Hashizume T, Hataya K et al (2005) CT of internal hernias. Radiographics 25(4):997-1015

5. Blachar A, Federle MP (2001) Bowel obstruction following liver transplantation: clinical and ct findings in 48 cases with emphasis on internal hernia. Radiology 218(2):384-388
6. Blachar A, Federle MP, Dodson SF (2001) Internal hernia: clinical and imaging findings in 17 patients with emphasis on CT criteria. Radiology 218(1):68-74

7. Blachar A, Federle MP, Pealer KM, Ikramuddin S, Schauer PR (2002) Gastrointestinal complications of laparoscopic Roux-en-Y gastric bypass surgery: clinical and imaging findings. Radiology 223(3):625-632

8. Hongo N, Mori H, Matsumoto S, Okino Y, Takaji R, Komatsu E (2011) Internal hernias after abdominal surgeries: MDCT features. Abdom Imaging 36(4):349-362

9. Sandrasegaran K, Maglinte DD, Lappas JC, Howard TJ (2005) Small-bowel complications of major gastrointestinal tract surgery. AJR Am J Roentgenol 185(3):671-681

10. Reddy SA, Yang C, McGinnis LA, Seggerman RE, Garza E, Ford KL 3rd (2007) Diagnosis of transmesocolic internal hernia as a complication of retrocolic gastric bypass: CT imaging criteria. AJR Am J Roentgenol 189(1):52-55

11. Iannuccilli JD, Grand D, Murphy BL, Evangelista P, Roye GD, Mayo-Smith W (2009) Sensitivity and specificity of eight CT signs in the preoperative diagnosis of internal mesenteric hernia following Roux-en-Y gastric bypass surgery. Clin Radiol 64(4):373-380

12. Balthazar EJ, Birnbaum BA, Megibow AJ, Gordon RB, Whelan CA, Hulnick DH (1992) Closed-loop and strangulating intestinal obstruction: CT signs. Radiology 185(3):769-775

13. Elsayes KM, Menias CO, Smullen TL, Platt JF (2007) Closed-loop small-bowel obstruction: diagnostic patterns by multidetector computed tomography. J Comput Assist Tomogr 31(5):697-701

14. Duda JB, Bhatt S, Dogra VS (2008) Utility of CT whirl sign in guiding management of small-bowel obstruction. AJR Am J Roentgenol 191(3):743-747

15. Wiesner W, Khurana B, Ji H, Ros PR (2003) CT of acute bowel ischemia. Radiology 226(3):635-650

16. Furukawa A, Kanasaki S, Kono N, Wakamiya M, Tanaka T, Takahashi $\mathrm{M}$ et al (2009) CT diagnosis of acute mesenteric ischemia from various causes. AJR Am J Roentgenol 192(2):408-416

17. Zalcman M, Sy M, Donckier V, Closset J, Gansbeke DV (2000) Helical CT signs in the diagnosis of intestinal ischemia in smallbowel obstruction. AJR Am J Roentgenol 175(6):1601-1607

18. Rha SE, Ha HK, Lee SH, Kim JH, Kim JK, Kim JH et al (2000) CT and MR imaging findings of bowel ischemia from various primary causes. Radiographics 20(1):29-42

19. Weinstein S, Osei-Bonsu S, Aslam R, Yee J (2013) Multidetector $\mathrm{CT}$ of the postoperative colon: review of normal appearances and common complications. Radiographics 33(2):515-532, Review

20. Cingi A, Cakir T, Sever A, Aktan AO (2006) Enterostomy site hernias: a clinical and computerized tomographic evaluation. Dis Colon Rectum 49(10):1559-1563

21. Moreno-Matias J, Serra-Aracil X, Darnell-Martin A, BombardoJunca J, Mora-Lopez L, Alcantara-Moral M et al (2009) The prevalence of parastomal hernia after formation of an end colostomy. A new clinico-radiological classification. Colorectal Dis 11(2):173177

22. Pilgrim CH, McIntyre R, Bailey M (2010) Prospective audit of parastomal hernia: prevalence and associated comorbidities. Dis Colon Rectum 53(1):71-76

23. Sandrasegaran K, Maglinte DD (2005) Imaging of small bowelrelated complications following major abdominal surgery. Eur $\mathrm{J}$ Radiol 53(3):374-386

24. Juan YH, Yu CY, Hsu HH, Huang GS, Chan DC, Liu CH et al (2011) Using multidetector-row CT for the diagnosis of afferent loop syndrome following gastroenterostomy reconstruction. Yonsei Med J 52(4):574-580

25. Jordan GL Jr (1971) Surgical management of postgastrectomy problems. Arch Surg 102(4):251-259

26. Wise SW (2000) Case 24: afferent loop syndrome. Radiology 216(1):142-145 
27. Gayer G, Barsuk D, Hertz M, Apter S, Zissin R (2002) CT diagnosis of afferent loop syndrome. Clin Radiol 57(9):835-839

28. Subhani M, Rizvon K, Mustacchia P (2012) Endoscopic evaluation of symptomatic patients following bariatric surgery: a literature review. Diagn Ther Endocrinol 753472. doi: $10.1155 / 2012 / 753472$

29. Mueller CL, Jackson TD, Swanson T, Pitzul K, Daigle C, Penner T et al (2013) Linear-stapled gastrojejunostomy with transverse handsewn enterotomy closure significantly reduces strictures for laparoscopic Roux-en-Y gastric bypass. Obes Surg 23(8):1302-1308

30. Hueting WE, Buskens E, van der Tweel I, Gooszen HG, van Laarhoven CJ (2005) Results and complications after ileal pouch anal anastomosis: a meta-analysis of 43 observational studies comprising 9,317 patients. Dig Surg 22(12):69-79
31. Broder JC, Tkacz JN, Anderson SW, Soto JA, Gupta A (2010) Ileal pouch-anal anastomosis surgery: imaging and intervention for post-operative complications. Radiographics 30(1):221-233

32. De Cruz P, Kamm MA, Prideaux L, Allen PB, Desmond PV (2012) Postoperative recurrent luminal Crohn's disease: a systematic review. Inflamm Bowel Dis 18(4):758-777

33. Expert Panel on Gastrointestinal Imaging (2011) ACR Appropriateness Criteria for Crohn disease American College of Radiology (ACR), Reston (VA): Available via http://www. guideline. gov/content.aspx?id=35137

34. Patel DR, Levine MS, Rubesin SE, Zafar H, Lev-Toaff AS (2013) Comparison of small bowel follow through and abdominal CT for detecting recurrent Crohn's disease in neoterminal ileum. Eur J Radiol 82(3):464-471 